\title{
openheart Current challenges in palliative care provision for heart failure in the UK: a survey on the perspectives of palliative care professionals
}

\author{
Mun Hong Cheang, ${ }^{1}$ Gabrielle Rose, ${ }^{2}$ Chi-Chi Cheung, ${ }^{3}$ Martin Thomas ${ }^{1}$
}

To cite: Cheang MH, Rose G, Cheung C-C, et al. Current challenges in palliative care provision for heart failure in the UK: a survey on the perspectives of palliative care professionals. Open Heart 2015;2:e000188. doi:10.1136/openhrt-2014000188

- Additional material is available. To view please visit the journal online (http://dx. doi.org/10.1136/openhrt2014-000188).

Received 28 August 2014 Revised 8 December 2014 Accepted 11 December 2014

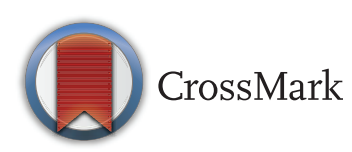

${ }^{1}$ Department of Heart Failure, The Heart Hospital, University College London Hospital, London, UK

${ }^{2}$ Camden Clinical

Commissioning Group, London, UK

${ }^{3}$ Camden, Islington ELiPSe, University College London Hospital \& HCA Palliative Care Service, CNWL NHS Foundation Trust, London, UK

Correspondence to Dr Mun Hong Cheang; mun.cheang@ucl.ac.uk

\section{ABSTRACT}

Objective: Palliative care (PC) in heart failure (HF) is beneficial and recommended in international $\mathrm{HF}$ guidelines. However, there is a perception that PC is underutilised in $\mathrm{HF}$ in the UK. This exploratory study aims to investigate, from a PC perspective, this perceived underutilisation and identify problems with current practice that may impact on the provision of PC in HF throughout the UK.

Methods: A prospective survey was electronically sent to PC doctors and nurses via the UK Association for Palliative Medicine and adult PC teams listed in the UK Hospice directory.

Results: We received 499 responses (42\%—PC consultants). Although PC provision for patients with HF was widespread, burden on PC services was low ( $47 \%$ received less than 10 referrals annually). While $\mathrm{PC}$ was acknowledged to have a role in end-stage HF, there were differing views about the optimal model of care. Levels of interdisciplinary collaboration $(58 \%)$ and mutual education (36\%) were low. There were frequent reports that end-of-life matters were not addressed by cardiology prior to PC referral. Moreover, $24 \%$ of respondents experienced difficulties with implantable cardioverter defibrillator deactivation. Conclusions: Low HF referrals despite widespread availability of $\mathrm{PC}$ services and insufficient efforts by cardiology to address PC issues may contribute to the perception that $\mathrm{PC}$ is underutilised in HF. The challenges facing $P C$ and $H F$ identified here need to be further investigated and addressed. These findings will hopefully promote awareness of $\mathrm{PC}$ issues in $\mathrm{HF}$ and encourage debate on how to improve PC support for this population.

\section{INTRODUCTION}

Heart failure (HF) is highly prevalent in the UK. ${ }^{1}$ In 2011, there were approximately 200000 cases. It is projected to increase with an ageing population. ${ }^{2}$ As in terminal cancers, HF has a heavy symptom burden and poor prognosis. ${ }^{3-5}$ Palliative care (PC) is defined as an approach that optimises quality of life (QOL) in life-threatening

\section{KEY QUESTIONS}

What is already known about this subject? Underutilisation of palliative care, unmet needs in heart failure, low referral numbers and other challenges related to palliative care issues in heart failure have been previously described in different countries and in some regions of the UK.

What does this study add?

This study provides a contemporary UK-wide overview of the perspectives of palliative professionals regarding significant issues related to palliative care and heart failure. In addition, this is the first study to document the difficulties associated with implantable cardioverter defibrillator (ICD) deactivation experienced by palliative professionals throughout the UK.

How might this impact on clinical practice? Our findings highlight the difficulties associated with provision of palliative care in heart failure (including ICD deactivation issues) from the perspective of palliative care professionals. By raising awareness of these challenges, it should encourage cardiologists to make efforts to improve current practice.

conditions. ${ }^{6}$ Prompt PC intervention alleviates symptoms and improves QOL in chronic disease and at end-of-life(EOL) ${ }^{7-9}$ In the $\mathrm{UK}$, it is delivered in the hospital, community or hospice setting by PC specialists or other healthcare professionals such as general practitioners (GPs) and HF specialists with adequate $\mathrm{PC}$ training. $\mathrm{PC}$ for $\mathrm{HF}$ is recommended in the latest European Society of Cardiology (ESC) and the UK's National Institute of Clinical Excellence HF guidelines. ${ }^{10} 11$

Numerous studies have documented various difficulties with $\mathrm{PC}$ provision in HF. These are mostly single centre studies, conducted in different countries or confined to regions in the UK. ${ }^{5}{ }^{12-14}$ It is not clear what 
the most significant challenges associated with the provision of PC in HF throughout the UK are. There is also a perception that PC services are underutilised in $\mathrm{HF}$ compared with cancer services. ${ }^{5}{ }^{12-14}$ Similarly, the nationwide significance of this problem and associated reasons are unknown.

This exploratory study aims to investigate the following questions from the perspective of the PC professional: (1) What are the reasons for the perceived underutilisation of PC services throughout the UK? (2) Are there any significant problems with current provision of $\mathrm{PC}$ for HF nationwide?

\section{METHODS}

We conducted a prospective survey of UK PC professionals. Four important themes were identified from the existing literature, ESC guidelines and the professional opinion of HF and PC members of this research group:

1. $\mathrm{PC}$ provision for HF services and the relative burden on $\mathrm{PC}$ resources;

2. Current practice and professional perception of the role of $\mathrm{PC}$ in $\mathrm{HF}$;

3. PC challenges specific to HF: implantable cardioverter defibrillator (ICD) deactivation and EOL discussions;

4. Interdisciplinary collaboration (which refers to various working arrangements that exist between cardiology and PC).

The survey consisted of 21 questions (see online supplementary file): 18 multiple choice questions (MCQs) with the option of entering free text in 7 of the 18 MCQs, 2 number entry questions and a final one asking for 'any other comments' (free text entry). Completion took 10-15 min. To assess the relative burden of patients with $\mathrm{HF}$ on $\mathrm{PC}$ services, respondents were requested to provide the number of $\mathrm{PC}$ patients and patients with $\mathrm{HF}$ currently under their care and to estimate the number of HF referrals they received in the preceding year. 'HF Burden' was defined as the number of patients with $\mathrm{HF}$ as a main diagnosis expressed as a percentage of the total number of patients currently under their care. In five MCQs, more than one response was allowed (indicated by 'tick all that apply' instruction). Respondents had the option to skip any question. The survey also collected information relating to the 'demographics' of our respondent population, that is, respondent's role (consultant or specialist nurse, etc), geographical locality, PC setting (hospital, hospice or community) and type of PC service they provided.

A provisional questionnaire was developed by one of the authors and subsequently reviewed by a group of $\mathrm{HF}$ and PC professionals (consisting of 5 doctors and nurses from 2 different institutions). Their suggestions were discussed by the research team and changes to wording, structure and arrangement of questionnaire were implemented to optimise the face and content validity. The final version was assessed for reliability by five PC professionals. The results were discussed and a consensus was reached on the final changes to the survey. SurveyMonkey, a web-based service, was used to create an online self-administered survey.

Our target population included consultants, nonconsultant doctors, specialist nurse practitioners and other nurses working in PC within the UK. In the covering letter, we explained the objective of the survey and requested that all members of our target population participate, even if they worked in the same PC service. They were also assured of anonymity and asked to fill in the survey just once. The survey was electronically sent out twice (3 months apart) to all members of the UK Association of Palliative Medicine. In addition, it was sent to all adult PC teams listed in the UK Hospice directory (http://www.hospiceuk.org) with the request that the recipient forward the email on to relevant team members. Our survey started in June 2013 and closed in December 2013. We received confirmation from the local ethics committee that ethics approval is not necessary for the conduct of this survey.

\section{Quantitative analysis}

The results were downloaded from SurveyMonkey onto an Excel spreadsheet format and exploratory data analysis was performed using Excel. Data were expressed as a median for continuous variables and frequencies for categorical data. As this study was designed to be exploratory, bivariate associations and inferential analysis were not undertaken.

\section{Qualitative analysis}

Free text was analysed by the framework approach. ${ }^{15}$ After familiarisation with the raw data, key themes were identified from the study objectives and issues raised by respondents. The raw data were organised according to the themes. Concepts and associations were highlighted and interpretations were subsequently made. The results were independently reviewed by each of the authors, discussed, and a conclusive interpretation was reached by consensus.

\section{RESULTS}

Eleven (2\%) of the 510 responses did not belong to the target population and were excluded. Respondents were distributed throughout all 19 regions of the UK (figure 1). Consultants accounted for $42 \%$ of responses (28\% clinical nurse specialists, $6 \%$ other PC nurses, 24\% nonconsultant doctors). Most were based mainly in a hospice (59\% hospice, $46 \%$ hospital, $21 \%$ community) and $18 \%$ worked in more than one site.

\section{PC provision for HF and the burden on services}

Almost all respondents offered PC services to patients with $\mathrm{HF}(97 \%)$. A range of PC services (which included hospice inpatient, day hospice, community and homecare, and hospital inpatient and outpatient) could be 




Figure 1 Number of consultants versus non-consultant doctors and nurses from different localities.

found in all regions of the UK. The minority (2\%) who did not offer services for HF gave no reason, were based in a cancer centre or mentioned having an alternative service (eg, joint PC clinics for patients without cancer).

Forty-seven per cent reported receiving less than 10 or no HF referrals in the preceding year while only $3 \%$ received more than 50 referrals (figure 2 ). Fifty per cent of participants were able to provide numbers of patients with HF and PC patients currently under their care. This cohort is well distributed throughout all regions of the UK (figure 3). The median 'HF burden' was 3\% of the total PC workload. Fifty-eight per cent of this cohort reported having a low HF burden (between 1\% and $10 \%$ ) while $27 \%$ reported having no patients with $\mathrm{HF}$ currently under their care. Similarly, low referral numbers were also reported in free text entries. Several respondents suggested that this may be due to underrecognition of PC needs by cardiologists, PC professionals and patients.

\section{Current practice and professional perception of the role of $\mathrm{PC}$ in $\mathrm{HF}$}

A minority of respondents (14\%) had specific HF referral criteria $(80 \%$ had no specific criteria and $6 \%$ 'did not know'). Table 1 lists criteria used by respondents. Breathlessness management pathway, symptom control guidelines and EOL pathways were commonly cited as treatment guidelines in HF. Subcutaneous diuretic and ICD deactivation guidelines were infrequently mentioned.

Almost all respondents (99\%) agreed that PC has a role in the management of severe or end-stage HF. The majority (more than $70 \%$ ) felt that end-stage HF (indicated by deterioration in symptoms, recurrent hospital admissions and EOL) was the most appropriate time for PC referral. Initial diagnosis or consideration of advanced HF management (ie, cardiac resynchronisation therapy, ICD or heart transplant) was not frequently cited as the 'most appropriate time for referral' ( $4 \%$ and $41 \%$, respectively).

Table 2 outlines the main themes that arose from relevant text responses. Despite unanimous agreement on the need for PC involvement in end-stage HF, there was a spectrum of views on the required level of involvement, as seen in comments below.

"If community matrons are involved there is often nothing additional for us to add until end of life," and

"...we should be seeing/helping more patients with end stage heart disease..."

\section{Difficulties with ICD deactivation}

Twenty-four per cent of respondents reported experiencing difficulties with ICD deactivation at EOL. These 


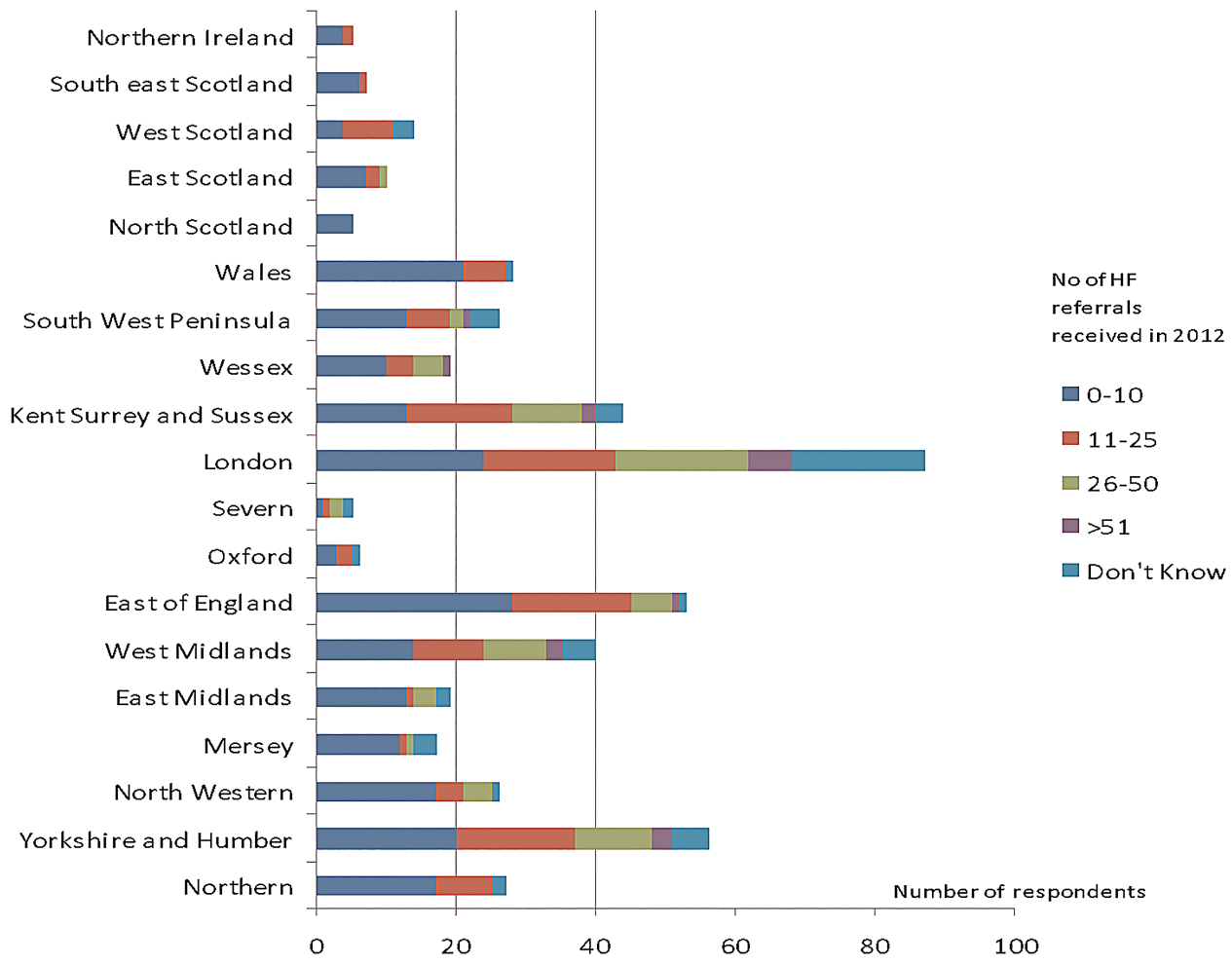

Figure 2 Reported number of heart failure (HF) referrals received in the previous year-according to locality.

respondents were distributed in all regions of the UK (apart from North Scotland). Notably, some respondents who reported 'no difficulties' admitted having had no prior or minimal experience with management of ICDs. The main difficulties associated with ICD deactivation can be summarised by three dominant themes, as outlined in table 3 .

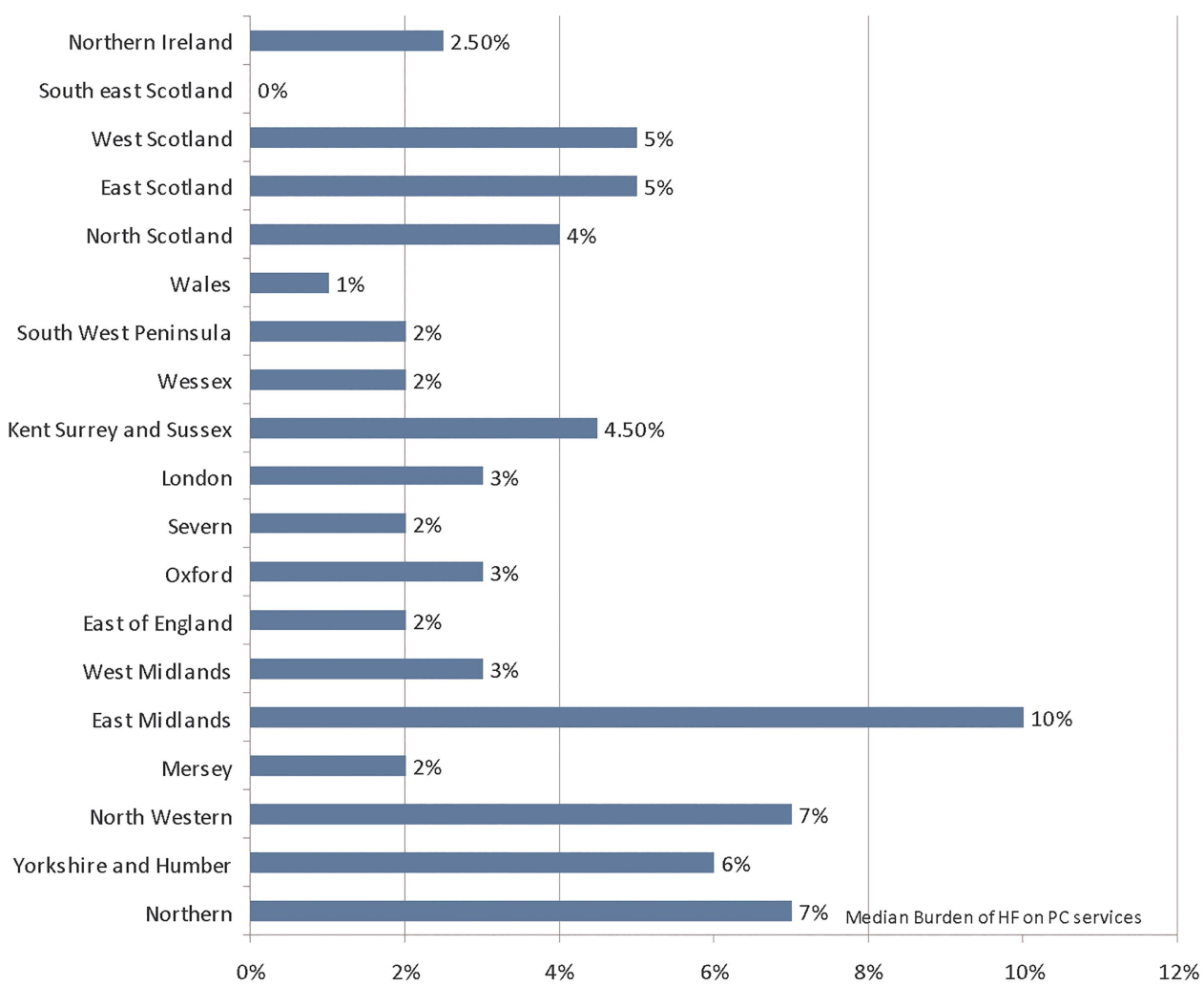

Figure 3 Median burden of heart failure (HF) on palliative care (PC) services (burden expressed as the percentage of patients with $\mathrm{HF}$ to the total number of PC patients currently under their care). 
Table 1 Referral criteria currently used

\begin{tabular}{ll}
\hline Commonly cited & Severity of HF \\
& Recurrent hospital admissions with \\
& decompensated HF \\
& Inappropriateness of further hospital \\
& admission \\
& When PC needs are not met by \\
& cardiology, including complex and \\
& persistent symptom control issues, \\
& psychosocial issues, EOL and ACP \\
& discussions \\
Less commonly & \\
cited & Exclusion of reversible causes \\
& Criteria based on Gold Standards \\
& Framework ${ }^{21}$-particularly prognostic \\
& indicators \\
& Generic (rather than disease \\
& specific) PC criteria
\end{tabular}

$\mathrm{ACP}$, advance care planning; $\mathrm{EOL}$, end-of-life; $\mathrm{HF}$, heart failure; $\mathrm{PC}$, palliative care.

Conversely, respondents in localities where an ICD deactivation policy had been set up reported significant improvements in service (particularly better timeliness and access to deactivation service or magnets during out-of-hours, in hospices or the community) and greater interdisciplinary cooperation.

\section{Other PC challenges in cardiology}

A large majority $(83 \%)$ of respondents reported that issues surrounding ICD deactivation and EOL care were seldom or never discussed by cardiology before making a PC referral. This problem was seen in all regions of the UK. There was broad agreement that further improvements to PC support in HF were needed, particularly in the home or community setting (although there were reports of good community support provided by some $\mathrm{HF}$ nurses). Other difficulties unique to $\mathrm{HF}$ are tabulated in table 4. Examples of efforts to improve PC service include new staff recruitment, active research and creating new pathways, working arrangements or treatment guidelines.

\section{Collaboration between cardiology and PC}

Interdisciplinary collaboration could be found in all regions of the UK and in all three PC settings. However, only $58 \%$ of all respondents reported having some form of collaboration. This figure increased to $71 \%$ when consultant responses were analysed in isolation (figure 4). 'Joint working', which refers to informal ad hoc working arrangements based on need, was most common $(71 \%)$. More formal organised collaborations such as preplanned multidisciplinary team meetings $(37 \%)$, steering/working groups $(21 \%)$ and mutual education organised between PC and HF (36\%) were less frequently observed. Nevertheless, several comments

Table 2 Role of PC in HF-main themes

Themes

\section{Examples of comments}

"The HF patients often have a very different journey from cancer patients...symptoms tend to be long standing and chronic" "There is a role for end of life management but l'm not sure if that role is specialist palliative care as we do it in other situations"

Uncertainty about optimal timing for PC involvement due to unpredictable prognostication

"Shared care" is preferred as HF team frequently continues involvement

PC's role is to "support" HF team in complex symptom management, ACP discussions and other issues related to $\mathrm{EOL}$

Some situations require $\mathrm{PC}$ teams to lead and initiate intervention
"It can be very difficult to know when to become involved with this client group"

"The most difficult problem we have with this group of patients is being able to assess that they really are end of life, as we do not have the resources to keep them on our case loads long term" "...unpredictability also makes people unsure when to refer"

“...treatment of the underlying condition remains important throughout; therefore the heart failure CNSs remaining involved is essential"

"Most of what we do is shared care rather than "transfer to""

“...role of palliative care teams...is supporting the cardiology teams to be more comfortable with complex communications especially around resuscitation, $\mathrm{ACP}$, etc"

"We often just give advice and leave them in the hands of the heart failure specialist nurses"

"Heart failure CNS(s) vary in their confidence to manage symptoms \& discussions surrounding EOLC issues \& we sometimes need to take a lead with this"

"...patients are reluctant to engage and cardiologists not sure when and how to do so"

ACP, advance care planning; CNS, Clinical Nurse Specialists; EOL, end-of-life; HF, heart failure; PC, palliative care. 
Table 3 Difficulties associated with implantable cardioverter defibrillator (ICD) deactivation-three main themes

Themes Examples of comments

\section{Reasons for limited access to ICD deactivation}

Poor out-of-hours access

Unavailable in the community or hospice

Excessive time delays

Lack of access to magnets

Insufficient education on ICD deactivation

Organisational difficulties

"Unable to deactivate out of hours"

"No availability of staff or a magnet to deactivate out of hours in a community hospital"

"Difficulty getting ICDs inactivated as outpatients or when hospice inpatient" "Patients in the community-Unable to access anyone to come out and deactivate in a dying patient"

"Delays, particularly in the community setting"

"Delays due to lack of defined process in the community"

"Unavailability of magnets for temporary deactivation"

"Needed to obtain magnet from CCU out of hours in order to deactivate ICD"

"Confusion around size of magnet needed"

"... difficult to arrange availability of magnet and to ensure education on how to use one"

"Access to technician support in the community—organised eventually but took lots of phone calls"

"No local policy to my knowledge"

"Took time to make contacts to arrange deactivation of ICD. Would be good to have regional flow charts on procedure for deactivation and contact numbers"

\section{Issues surrounding ICD deactivation that require improvement}

Decision-making on ICD deactivation

Advance care planning: pre-empting ICD deactivation

Communication issues related to ICD deactivation
"Cardiology team reluctant to take the lead on decision-making"

"Electrophysiology services unwilling to have conversations and make decisions (regarding) turning off ICDs"

"Not thought about early enough, unable to deactivate in time"

"Lack of forward planning at times in the community"

"Prior discussions on deactivation-that have not been had early enough"

"Problems more associated with difficult communication issues"

"Medical team reluctant to discuss with patient"

"Need to ensure discussion about deactivation occur at insertion"

"These discussions take place at the point at which they are inserted but are usually forgotten by the time they come into focus"

"Patients not knowing they will need to be deactivated"

\section{Patient-related barriers to deactivation}

Patient reluctance or refusal to deactivate their ICD
"Patient was against having ICD deactivated"

"Patient was reluctant to the idea of turning the device off"

"...patient did not want it deactivated but his family did"

CCU, Coronary Care Unit.

reveal that informal working arrangements in a supportive environment can also be productive. Other examples of current collaborative models of care include joint (clinic/home) consultations, jointly organised patient support groups, HF rehabilitation programmes in hospices and assimilating PC professionals into HF teams.

Although there were some reports of good existing cooperation, several respondents expressed the desire to foster closer links with cardiology. There was recognition that close working relationships encouraged appropriate referrals and improved mutual education, patient outcomes and staff satisfaction.

\section{DISCUSSIONS AND LIMITATIONS}

Although this study involved many respondents widely distributed throughout the UK, as in all surveys, a degree of sampling bias is to be expected. First, most respondents were based in a hospital or hospice setting. Therefore, while it is fairly common for PC professionals in the UK to work in hospice and community, our findings do not adequately reflect the unique issues associated with community $\mathrm{PC}$ provision. Second, a limitation associated with our method of distribution is the fact that the survey responder rate (which is a conventional marker of sampling bias) could not be calculated. Nonetheless, we have estimated a consultant 
Table 4 Other difficulties unique to palliative care (PC) in heart failure (HF)

\section{Themes}

Cardiologists are unfamiliar with identifying and managing palliative needs of patients with HF

\section{Observations of reluctance by cardiologists to engage with} PC profession

Observations of patient reluctance to engage with PC profession for various reasons (eg, unaware of severity of their condition and stigma associated with use of PC)

Insufficient PC resources to cope with additional workload

A need and desire by PC professionals to improve training in relevant $\mathrm{PC}$ skills for cardiology and vice versa for $\mathrm{PC}$

\section{Examples of comments}

"Advance care planning is the main challenge we have... cardiologists not sure when and how to do so" "...reluctance to refer patients because discussions (regarding prognosis) have never happened..."

"Some heart failure clinicians easier to engage than others" "Having a cardiologist that 'doesn't do palliative' can make it more difficult to give help/advice to both patients and other team members"

"The main issue often...(is) prognostication and the patients' perception of how ill they potentially are"

"...HF patients often have a very different journey from cancer patients and the stigma surrounding palliative care and cancer at times persists"

"...we have not had success in persuading patients to have PC assessment"

“...should be more involved with heart failure patients...the issue is how to do this most effectively with an already over stretched palliative care service...needs to be investment into palliative care services to provide this"

"...we do not have the resources to keep them (HF patients) on our case loads long term..."

"Two of the greatest barriers to good care by palliative services for patients with heart failure is...the limited knowledge that palliative specialist doctors and nurses have into the current best management of heart failure (esp. cardiac drugs and interventions such as biventricular pacemakers) not just to prolong life but to best manage symptoms caused by fluid overload and weak cardiac function..."

"....in my experience the reluctance (by the cardiology team) to provide this (PC) is...lack of knowledge in symptom control and complex communication but with support from palliative care teams and education this can be improved" "General lack of confidence amongst PC CNSs around heart failure as opposed to malignancy" survey responder rate of $42 \%$ from the 2012 Royal College of Physicians workforce census ${ }^{16}$ (210 consultant responses vs 502 known PC consultants in the UK).

It is worth noting that this exploratory study was mainly designed to identify reasons for the perceived underutilisation of PC services and to detect any problems currently associated with present practice. Conducting an accurate UK-wide census of PC services for $\mathrm{HF}$ was not our study's objective. Hence, while our results may indicate availability of PC services, it should neither be interpreted as a definitive assessment of provision in $\mathrm{HF}$ nor as a proportional representation of the views of PC professionals. Furthermore, the strength of signal in the qualitative analyses was not determined given the exploratory design of this survey. The deliberate decision to principally target PC professionals in this exploratory survey is based on the recognition that they are more likely to recognise unaddressed PC needs in the HF population. Thus, our findings offer a PC's perspective into the current challenges facing $\mathrm{PC}$ in $\mathrm{HF}$. It will be interesting and valuable for future studies to further investigate the issues outlined in this study by evaluating the alternate perspectives of HF professionals, GPs (who are another source of community HF referrals to PC services in the $\mathrm{UK}^{17}$ ) and patients.

This survey reveals that although a range of $\mathrm{PC}$ services for $\mathrm{HF}$ is offered throughout the $\mathrm{UK}, \mathrm{HF}$ referral numbers (and consequently burden on PC services) remain low. In addition, two observations were noted: an impression that cardiologists were under-referring and reluctant to engage with $\mathrm{PC}$. The frequency of these observations is undetermined. Low HF burden or referral numbers and infrequent PC referrals made by cardiologists have been similarly documented in Ireland, ${ }^{18}$ England $^{12}$ and America, ${ }^{14}$ respectively. Admittedly, not all patients with $\mathrm{HF}$ require specialist $\mathrm{PC}$ input and the level of PC need (hence requirement for formal referral) fluctuates with time. Moreover, reports of specialist PC 
Non-consultant Doctors and Nurses

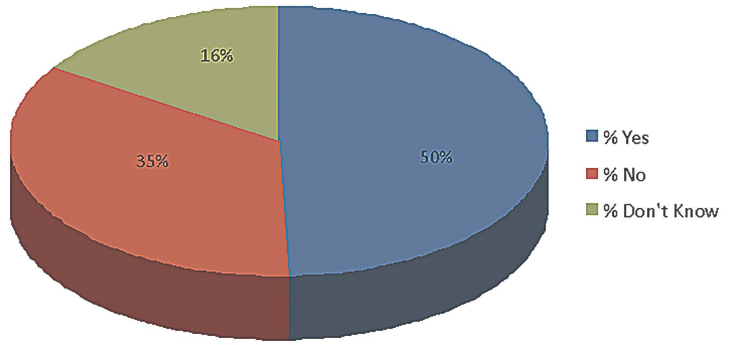

Consultant \& Clinical Lead

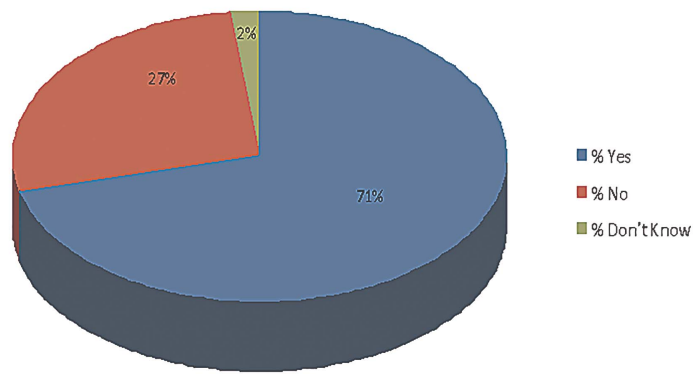

All Respondents

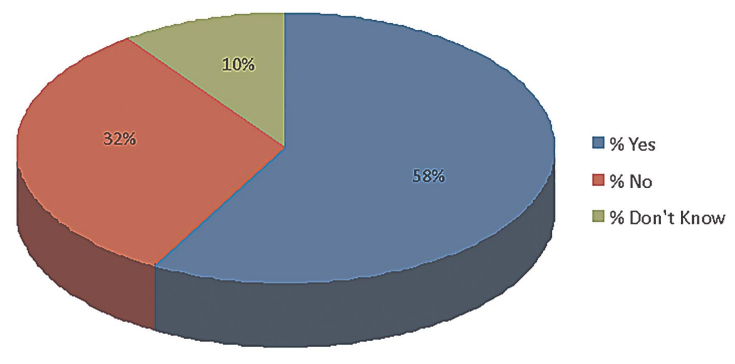

Figure 4 Reported rates of collaboration in three different cohorts-all respondents, consultants and non-consultant staff.

providing a 'supportive' and advisory role also means some of the burden is shared between HF and PC teams. Despite that, the low referral numbers do appear to be at odds with high HF prevalence. Recurrent observations by PC professionals of reluctance or unfamiliarity within cardiology to engage with and address $\mathrm{PC}$ issues with patients (including opening EOL and ICD deactivation discussions) corresponds with the published literature. ${ }^{19}{ }^{20}$ It is likely that these factors contribute to the perception of $\mathrm{PC}$ underutilisation in HF.

As terminal diseases often tend to have similar PC needs at EOL, it is unsurprising that the use of generic referral criteria and treatment guidelines is common practice. However, the significance of the infrequent use of HF-specific criteria and guidelines is unclear. In addition, $42 \%$ of respondents did not cite "consideration of advanced HF management" as "most appropriate time for referral" despite it being indicative of increasing symptom burden and PC need. ${ }^{6}$ Further investigation is needed to ascertain if these observations indicate insufficient effort by PC services to cater for disease-specific needs, unfamiliarity with disease trajectory or an alternative explanation. Indeed, the overall impression from this survey is that further improvement in PC support for patients with HF is needed in the UK. Nevertheless, the key question is whether PC needs in HF are currently inadequately addressed nationally. If so, this could be due to underrecognition of HF needs by both specialties.

Despite broad acknowledgement of the value of PC in end-stage or severe $\mathrm{HF}$, there was divergence in views on the level and timing of PC input. PC needs are often predicated on whether a patient is nearing EOL. Tools such as the Gold Standards Framework (GSF) Prognostic Indicator guidance ${ }^{21}$ and the Supportive and Palliative Care Indicator Tools (SPICT), ${ }^{22}$ have been developed through research and collaboration to help identify patients approaching EOL in order to meet clinical need. Indeed, the referral criteria cited by respondents are supported by the GSF and SPICT tools. However, the clinical trajectory in $\mathrm{HF}$ is inherently unpredictable. ${ }^{23}$ Given that predicting PC needs with clinical indicators is fraught with difficulties, professional uncertainty regarding the optimal time ${ }^{20}$ to initiate PC needs assessment and discussion is unsurprising. This has contributed to the growing opinion that a better option may be to introduce PC at an earlier stage to run in tandem with conventional HF treatment such that PC provision may be more responsive to the individuals' fluctuating needs throughout the disease journey. ${ }^{24}$

There were markedly different views among PC professionals on the optimal model of care to cope with ever increasing HF demand. It is certainly encouraging to see different interdisciplinary working arrangements evolving to suit the skills and enthusiasm of local teams and resource availability in order to meet local demand. However, more research into optimum models of PC delivery is warranted.

The relatively low level of interdisciplinary collaboration reported by PC professionals is consistent with a 2004 survey $^{12}$ conducted (in England) by Gibbs et al (58\% in present study vs $59 \%$ in 2004). Furthermore, the disparity in reported levels of collaboration by consultants versus non-consultant staff may indicate less involvement of non-consultant staff in interdisciplinary cooperation and merits further exploration. Despite 
that, services that made concerted interdisciplinary efforts to deal with all PC issues reported improved outcomes. This is in agreement with published evidence. ${ }^{59}$ There is certainly a need ${ }^{25}$ and desire among PC professionals (reflected in comments) to develop interdisciplinary education and improve mutual skills.

When managed poorly, ICD deactivation can cause significant distress to the patient and family. ${ }^{26}$ Disappointingly, the 24\% who experienced ICD difficulties were distributed in nearly all regions of the UK. Moreover, this figure is probably an underestimate as low referral rates could be masking the true extent of the problem. It is worth noting that many of the listed factors contributing to ICD difficulties are related to poor service organisation. This problem is not unique to the UK, as suboptimal provision of ICD deactivation service in US hospices was also documented in $2010 .^{27}$ In addition, observations of patient reluctance with deactivation and to engage with $\mathrm{PC}$ emphasises the importance of early initiation of frank PC discussions.

We have presented a UK-wide overview of significant challenges currently facing PC and HF from a PC perspective. This is also the first study, to our knowledge, to document the difficulties surrounding ICD deactivation throughout the UK. It is disappointing to find that difficulties still persist in PC provision for $\mathrm{HF}$ in 2014. We hope that our findings will reinvigorate the debate on PC in HF among cardiologists, particularly on the following issues: reassessment of contemporary PC needs as new HF therapies emerge, optimal timing for initiating PC discussions or needs assessment and ideal interdisciplinary working arrangements to deliver efficient care. The fact remains that cardiologists are often the gatekeepers to PC services for the patient with HF. Therefore, improvement of $\mathrm{PC}$ provision in HF must first start with raising awareness of its benefits among cardiologists and encouraging interdisciplinary dialogue.

Acknowledgements The authors would like to thank all the participants of the survey for their valuable input.

Contributors All the authors have contributed to the planning and analysis of this study. MHC and GR have been mainly in charge of the conduct of the study.

Competing interests None.

Provenance and peer review Not commissioned; externally peer reviewed.

Data sharing statement All the data from this study are only available to the authors listed in this paper.

Open Access This is an Open Access article distributed in accordance with the Creative Commons Attribution Non Commercial (CC BY-NC 4.0) license, which permits others to distribute, remix, adapt, build upon this work noncommercially, and license their derivative works on different terms, provided the original work is properly cited and the use is non-commercial. See: http:// creativecommons.org/licenses/by-nc/4.0/

\section{REFERENCES}

1. British Heart Foundation. Prevalence. http://www.bhf.org.uk/research/ heart-statistics/morbidity/prevalence.aspx (accessed 8 Apr 2014).

2. Blinderman CD, Homel P, Billings JA, et al. Symptom distress and quality of life in patients with advanced congestive heart failure. J Pain Symptom Manage 2008;35:594-603.
3. Solano JP, Gomes B, Higginson IJ. A comparison of symptom prevalence in far advanced cancer, AIDS, heart disease, chronic obstructive pulmonary disease and renal disease. J Pain Symptom Manage 2006;31:58-69.

4. NICOR: National Institute for Cardiovascular Outcomes Research. National clinical audit: Heart Failure Annual report 2012/13. https:// www.ucl.ac.uk/nicor/audits/heartfailure/reports (accessed 8 Apr 2014).

5. O'Leary N. The comparative palliative care needs of those with heart failure and cancer patients. Curr Opin Support Palliat Care 2009;3:241-6.

6. Jaarsma T, Beattie JM, Ryder M, et al. Advanced Heart Failure Study Group of the HFA of the ESC. Palliative care in heart failure: a position statement from the palliative care workshop of the Heart Failure Association of the European Society of Cardiology. Eur $J$ Heart Fail 2009;11:433-43.

7. Lorenz KA, Lynn J, Dy SM, et al. Evidence for improving palliative care at the end of life: a systematic review. Ann Intern Med 2008;148:147-59.

8. Gomes B, Calanzani N, Curiale V, et al. Effectiveness and cost-effectiveness of home palliative care services for adults with advanced illness and their caregivers. Cochrane Database Syst Rev 2013:6:CD007760.

9. Bailey FA, Burgio KL, Woodby LL, et al. Improving processes of hospital care during the last hours of life. Arch Intern Med 2005;165:1722-7.

10. European Society of Cardiology. ESC Clinical Practice Guidelines: Acute and Chronic Heart Failure. http://www.escardio.org/ guidelines-surveys/esc-guidelines/Pages/acute-chronic-heart-failure. aspx (accessed 8 Apr 2014).

11. National Institute for Health and Care Excellence. Chronic heart failure: management of chronic heart failure in adults in primary and secondary care. http://www.nice.org.uk/guidance/cg108/resources/ guidance-chronic-heart-failure-pdf (accessed 24 Jul 2014).

12. Gibbs LM, Khatri AK, Gibbs JS. Survey of specialist palliative care and heart failure: September 2004. Palliat Med 2006;20:603-9.

13. Anderson $\mathrm{H}$, Ward C, Eardley A, et al. The concerns of patients under palliative care and a heart failure clinic are not being met. Palliat Med 2001;15:279-86.

14. Riegel B, Moser DK, Powell M, et al. Nonpharmacologic care by heart failure experts. J Card Fail 2006;12:149-53.

15. Pope C, Ziebland S, Mays N. Qualitative research in health care. Analysing qualitative data. BMJ 2000;320:114-16.

16. Federation of Royal Colleges of Physicians' annual census of consultant physicians and medical registrars in the UK 2012. https://www.rcplondon. ac.uk/resources/2012-census-summary (accessed 24 Jul 2014).

17. Oishi A, Murtagh FE. The challenges of uncertainty and interprofessional collaboration in palliative care for non-cancer patients in the community: a systematic review of views from patients, carers and health-care professionals. Palliat Med 2014:28:1081-98.

18. O'Leary N, Tiernan E. Survey of specialist palliative care services for noncancer patients in Ireland and perceived barriers. Palliat Med 2008;22:77-83.

19. Barclay S, Momen N, Case-Upton S, et al. End-of-life care conversations with heart failure patients: a systematic literature review and narrative synthesis. Br J Gen Pract 2011;61:e49-62.

20. Kavalieratos D, Mitchell EM, Carey TS, et al. "Not the "grim reaper service'": an assessment of provider knowledge, attitudes, and perceptions regarding palliative care referral barriers in heart failure. J Am Heart Assoc 2014;3:e000544.

21. The Gold Standards Framework. Library, Tools \& ResourcesPrognostic Indicator Guidance. http://www.goldstandardsframework.org. uk/cd-content/uploads/files/General\%20Files/Prognostic\%20Indicator\% 20Guidance\%20October\%202011.pdf (accessed 8 Apr 2014).

22. Supportive and Palliative Care Indicators Tool (SPICT). http://www. spict.org.uk (accessed 8 Apr 2014).

23. Haga K, Murray S, Reid J, et al. Identifying community based chronic heart failure patients in the last year of life: a comparison of the Gold Standards Framework Prognostic Indicator Guide and the Seattle Heart Failure Model. Heart 2012;98:579-83.

24. Gadoud A, Jenkins SM, Hogg KJ. Palliative care for people with heart failure: summary of current evidence and future direction. Palliat Med 2013;27:822-8.

25. Green E, Gardiner C, Gott M, et al. Exploring the extent of communication surrounding transitions to palliative care in heart failure: the perspectives of health care professionals. $J$ Palliat Care 2011:27:107-16.

26. Fromme EK, Stewart TL, Jeppesen M, et al. Adverse experiences with implantable defibrillators in Oregon hospices. Am J Hosp Palliat Care 2011;28:304-9.

27. Goldstein N, Carlson M, Livote E, et al. Brief communication: management of implantable cardioverter-defibrillators in hospice: a nationwide survey. Ann Intern Med 2010;152:296-9. 